\title{
Transient Stability Assessment of Power System Based on Support Vector Machine
}

\author{
Shengyong Ye Yongkang Zheng Qingquan Qian \\ School of Electrical Engineering, Southwest Jiaotong University, Chengdu 610031, P. R. China
}

\begin{abstract}
Machine learning methods are promising tools for transient stability assessment (TSA) of power system. Support vector machine (SVM) is used to assess the transient stability of power system after faults occur on transmission lines. Single machine attributes is studied as inputs of the SVM classifier. Experimental results in IEEE 50-generator test system shows that, attributes of single machine with small inertia coefficient are effective in TSA, and the SVM classifier with RBF kernel using these single machine attributes can achieve satisfying classification accuracy.
\end{abstract}

Keywords: Transient stability assessment, Support vector machines, Single machine attributes

\section{Introduction}

Electric power systems are large-scale non-linear systems where there are many kinds of stability problems. One of them is transient stability, which is defined as the ability of a power system to maintain synchronism after severe disturbances. The purpose of transient stability assessment (TSA) is to determine if the contingency may cause power system into angle instability, that is, to predict whether the power system could maintain synchronous operation of generators when subjected to large disturbances such as faults, load loss, capacity loss, etc.

One of conventional methods used for TSA is the time-domain numerical simulation. This method consists of simulating during and post-fault behaviors of the system for a given disturbance, observing its electromechanical angular swings during a few seconds. It is usually used to estimate stability status and to provide detailed operation information of the faulted systems as a benchmark. However, the simulation method is infeasible for on-line TSA mainly due to its time-consuming computation.

Direct method is another conventional approach used in TSA, which replaces the numerical integration of the post-fault system equations by a stability criterion [1]. Although it is attractive for TSA, it has always given conservative results since its deficiency.

Due to the limitations of time-domain and direct methods, there have been great interests in applying machine learning methods, which are promising for on-line application. [2] summarized experiences in applying artificial neural network (ANN) to TSA. ANN requires long-time training process and the selection of the number of neurons in hidden layer is usually determined by a trial. Decision tree has been studied to be used in TSA [3], [4]. These methods provide mapping between the system variables and status with the aid of time-domain simulations.

With support vector machine (SVM) introduced by Vapnik and his co-worker [5], many TSA applications based on SVM have come forth [7], [8]. [7] discussed the SVC with polynomial kernel using 224 input variables. A $v$-SVM with thirteen features is applied in TSA [8].

A SVM using single machine attributes is proposed in this paper. Case study using IEEE 50generator test system was presented to illustrate the proposed method. The paper is organized as follows. Section 2 provides a simple review of TSA and a set of single machine attributes. We demonstrate the techniques in Section 3. In Section 4 we present an experimental comparison among ANN, Decision tree and SVM, since the first two are always used in TSA [9]. In Section 5 we discuss the results. Finally, the conclusion of our research is in Section 6.

\section{Single machine attributes}

As a benchmark of TSA, time-domain simulation is given as follows. For a given disturbance, the simulation program alternately solves the nonlinear equations representing the dynamics of generators, and algebraic power-flow equations representing the network. For illustration, consider a multi-machine system. The motion of the ith generator is described as follows: 


$$
\begin{aligned}
\frac{d \delta_{i}}{d t} & =\omega_{i}-\omega_{n}, \\
M_{i} \frac{d \omega_{i}}{d t} & =P_{m i}-P_{e i}, \quad i=1,2, \ldots, n .
\end{aligned}
$$

where

$\delta_{i}=$ rotor angle of the ith generator;

$\omega_{i}=$ the speed of the ith generator;

$\omega_{n}=$ the synchronous speed of the ith generator;

$M_{i}=$ inertia coefficient of the ith generator;

$P_{m i}=$ mechanical power input of the ith generator

$P_{e i}=$ electric power output of the ith generator.

For example, in IEEE 50-generator system, after a few seconds simulation, power angles vs. time are shown as Fig.1 (stable) and Fig.2 (unstable) for two different disturbances.

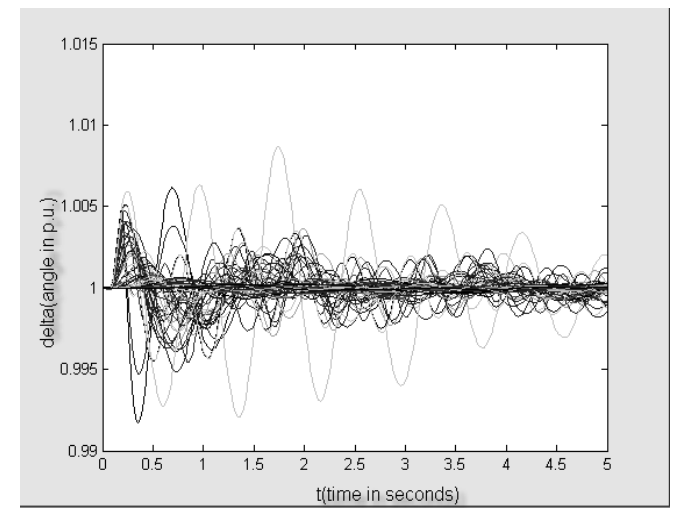

Fig. 1: Time-domain simulation results for stable state.

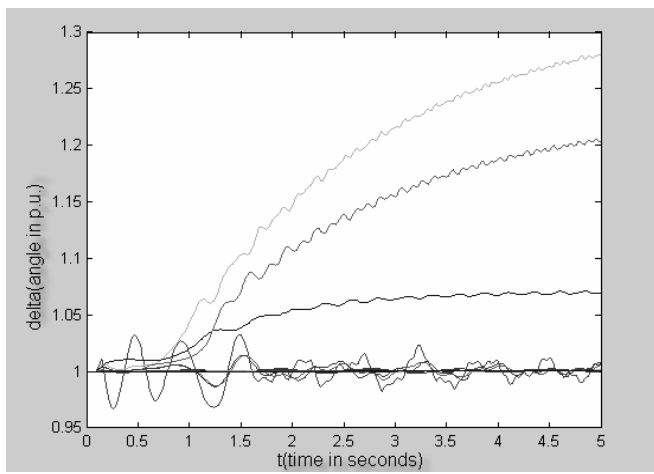

Fig. 2: Time-domain simulation results for unstable state.

It is difficult to decide attributes as inputs of the machine learning. Generally, the post-fault variables of generator rotors are first choices for TSA. Inputs comprising variables of all the generators make large input dimension because power systems are very large. Some researchers propose attributes independent of the size of power system[10].
In this paper, we try to use single machine attributes as inputs. Seven variables of a generator are chosen, such as machine angle, machine speed, machine terminal voltage, electrical active output power, electrical reactive output power, the derivatives of machine angle and speed to time.

Twenty-eight attributes, which were the above variables in four different moments, composed the input space. One moment was during the fault, others were after the fault.

\section{Support vector machine classifier}

\subsection{Introduction to SVM}

SVM is based on theoretical results from the statistical learning theory. It is a new and promising technique for data classification and regression. In this section, we briefly introduce support vector machine classifier which can be used for TSA.

Given training data set $\left(\boldsymbol{x}_{i}, y_{i}\right), i=1, \ldots, l$ where $\boldsymbol{x}_{i} \in R^{n}$ and $y_{i} \in\{-1,1\}$, the aim of SVM classifier is to separate two kinds of data in high feature space by constructing an optimal hyperplane. SVM classifier solves following prime problem:

$$
\begin{array}{ll}
\min _{w, b, \xi} & \frac{1}{2} \boldsymbol{w}^{T} \boldsymbol{w}+C \sum_{i=1}^{l} \xi_{i} \\
\text { s.t. } & y_{i}\left(\boldsymbol{w}^{T} \phi\left(\boldsymbol{x}_{i}\right)+b\right) \geq 1-\xi_{i} \\
& \xi_{i} \geq 0, i=1, \ldots, l .
\end{array}
$$

where $\boldsymbol{w}$ is weight vector of the hyperplane, $C>0$ is penalty parameter proportional to the amount of constraint violation, $\xi$ is slack variable, $\phi(\cdot)$ is a mapping from input space to feature space, and $b$ is threshold .

The dual of (2) is

$$
\begin{array}{ll}
\min _{\alpha} & \frac{1}{2} \boldsymbol{\alpha}^{T} \mathbf{Q} \boldsymbol{\alpha}-e^{T} \boldsymbol{\alpha} \\
\text { s.t. } & y^{T} \boldsymbol{\alpha}=0 \\
& 0 \leq \alpha_{i} \leq C, \quad i=1, \ldots, l
\end{array}
$$

where $\alpha$ is a vector of $l$ variables, $\boldsymbol{e}$ is the vector of all ones.

The most frequently used kernel functions are as follows.

Linear kernel:

$$
K\left(\boldsymbol{x}_{i}, \boldsymbol{x}_{j}\right)=\boldsymbol{x}_{i}^{T} \boldsymbol{x}_{j} .
$$

Polynomial kernel:

$$
K\left(\boldsymbol{x}_{i}, \boldsymbol{x}_{j}\right)=\left(\gamma \boldsymbol{x}_{i}^{T} \boldsymbol{x}_{j}+r\right), \quad \gamma>0 .
$$


Radial basis function kernel:

$$
K\left(\boldsymbol{x}_{i}, \boldsymbol{x}_{j}\right)=\exp \left(-\gamma\left\|\boldsymbol{x}_{i}-\boldsymbol{x}_{j}\right\|^{2}\right), \quad \gamma>0 .
$$

Here, $\gamma$ and $r$ are kernel parameters.

\subsection{Model selection in SVM classifier}

Upon the data set prepared using time-domain simulation, SVM classifier model was built for TSA. When training an SVM classifier model, there are some parameters to tune. They would influence the performance of the SVM classifier model. kernel parameters and cost of error $\mathrm{C}$ should be decided before SVM classifier training. The process to search optimal values of these parameters is model selection.

In our experiments, we considered the radial basis function (RBF) kernel. Note that $\gamma$ is a tunable parameter associated with the RBF function. Thus, $\gamma$ and $\mathrm{C}$ are two parameters needed searched in model selection process[11]. To do this, we divided the training data into two sets. One of them was used to train a model while the other, called the validation set, was used to evaluate the model. We used grid search technique to find the optimal values. The search result is shown in Fig.3.

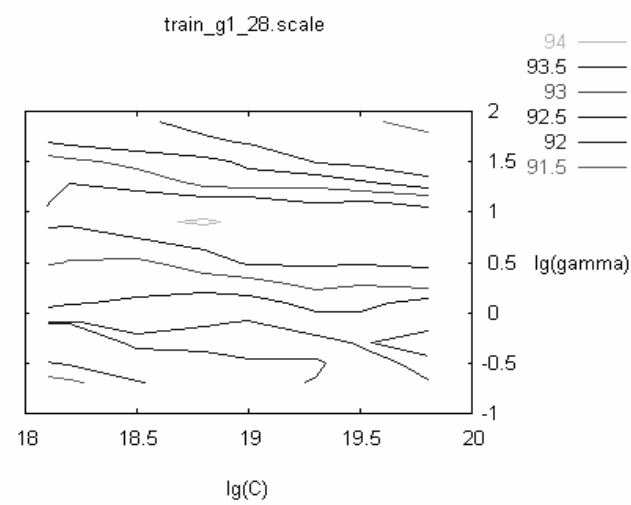

Fig. 3: Grid search for proper parameters.

We built the SVM classifier model using the proper $\mathrm{C}$ and $\gamma$. SMO was used to train the SVC model. When the training process finished, the SVM classifier's performance was assessed by test set.

For comparison[12], two classifiers based on ANN and Decision tree were trained using the same training set. The ANN used in this study is the MLP type. It consisted of three layers. The learning rule was Momentum, which provided the gradient with some inertia, while the amount of inertia was dictated by the momentum parameter. Decision tree was generated by C4.5. The confidence factor used for pruning was set as 0.25 , and the minimum number of instances per leaf was two.

\section{Case study}

\subsection{Test system}

IEEE 50-generator test system [13] was used to test the validity of the proposed method, with some generator was modified. There are seven generators (Generator 1 to 6, 23) based on model including subtransient effect and 43 generators based on classical model. A large amount of transient stability simulations were carried out to obtain training and test sets. Three-phase short-circuit faults were created at instance $0.1 \mathrm{~s}$ and cleared at $0.2 \mathrm{~s}$ and $0.25 \mathrm{~s}$.

Under $90 \%, 100 \%, 110 \%$ and $120 \%$ of the basic load conditions, there were 1812 examples created for every generator. The attribute vectors were acquired from those examples. These vectors were characterized as stable and unstable.

\subsection{Result}

To every generator, 1196 examples were assigned as training set, and 616 ones comprised test set. Using grid search technique, the kernel parameter $\gamma$ and the tradeoff parameter $\mathrm{C}$ of every classifiers were optimized by grid search. The correct classification rate using every generator attributes is shown in Fig.4, where $\mathrm{H}$ is inertia coefficient of generator. The range of $\mathrm{H}$ is from 0.7668 to $2210 \mathrm{~s}$.

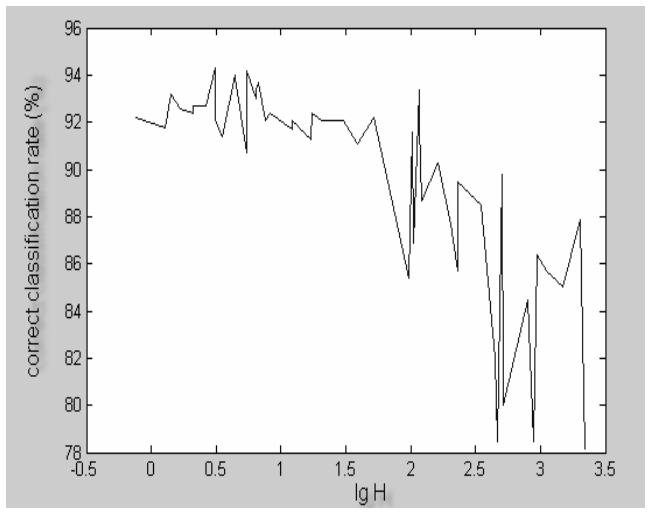

Fig. 4: Correct classification rates of classifiers using single generator attributes as inputs. 
As to the seven generators, the performances of their attributes as SVM classifier inputs are shown in Table 1.

\begin{tabular}{|c|c|c|c|}
\hline $\begin{array}{c}\text { Generator } \\
\text { number }\end{array}$ & $\begin{array}{c}\text { Inertia } \\
\text { coefficient } \\
(\mathrm{s})\end{array}$ & $(\mathrm{C}, \gamma)$ & $\begin{array}{c}\text { Correct } \\
\text { rate } \\
(\%)\end{array}$ \\
\hline 1 & 6.47 & $(131072,8)$ & 93.99 \\
\hline 2 & 3.14 & $(256,16)$ & 94.32 \\
\hline 3 & 4.43 & $(131072,1)$ & 94.00 \\
\hline 4 & 4.43 & $(512,2)$ & 93.84 \\
\hline 5 & 6.47 & $(32768,2)$ & 93.35 \\
\hline 6 & 3.14 & $(256,16)$ & 92.05 \\
\hline 23 & 3.53 & $(8192,32)$ & 91.41 \\
\hline
\end{tabular}

Table 1: Performances of seven generators' attributes as SVM classifier inputs.

For comparison with traditional classifiers, we chose Generator 1's attributes as inputs. After training, models of three classifications were checked in test set. The performances are shown in Table 2. All the computations presented in this paper were performed on Intel Celeron, $1.50 \mathrm{GHz}$, PC.

\begin{tabular}{|c|c|c|c|}
\hline $\begin{array}{c}\text { Machine } \\
\text { learning } \\
\text { classifiers }\end{array}$ & $\begin{array}{c}\text { Training } \\
\text { time } \\
\text { (s) }\end{array}$ & $\begin{array}{c}\text { Correct } \\
\text { rate } \\
(\%)\end{array}$ & $\begin{array}{c}\text { Kappa } \\
\text { statistic }\end{array}$ \\
\hline MLP & 100.76 & 91.07 & 0.77 \\
\hline Tree & 0.40 & 92.53 & 0.82 \\
\hline RBF-SVM & 3.15 & 93.99 & 0.85 \\
\hline
\end{tabular}

Table 2: Performances of three classifiers on test set.

\section{Discussion}

In the transient stability assessment using machine learning method, the key step is the selection of system variables. In power system simulation, generators with detailed model provide sufficient information in power system stability and control. We chose single machine variables as inputs rather than all machines or abstract attributes. The single machine attributes we proposed can effectively predict the system state in transient stability assessment. The attributes of generators with smaller inertia coefficient can give satisfying results. As shown in Fig.4, correct classification rates decrease with inertia coefficient increasing.

Model selection is another key problem in SVM. Kernel parameters and $\mathrm{C}$ should be proper. Otherwise, the classifier showed poor performance. We used simple grid search to find optimal parameters. The reason is that exhaustive parameter search could find optimal values. In our experiment, we used other search techniques, but the result was unsatisfying.

There are some suggestions for improvement in our study. The first is using proper feature selection to increase the correct classification rate and computing speed. Another is using other kernel function to improve the performance of SVM classifier.

\section{Conclusions}

We have presented single machine attributes as inputs of SVM classifier in assessing transient stability of power system. Extensive testing was performed on the IEEE 50-generator test system under various loading conditions. The attributes of machines with smaller inertia coefficients showed better performance. Based on the theory of statistical learning, SVM classifier shows better performance than traditional methods in TSA.

\section{References}

[1] M. Pavella, D. Ernst, D. Ruiz-Vega, Transient stability of power systems: a unified approach to assessment and control. Boston: Kluwer cademic Publishers, 2000.

[2] Y. Mansour, E. Vaahedi, M. A. El-Sharkawi, Dynamic security contingency screening and ranking using neural networks. Neural Networks, IEEE Transactions on, 8: 942-950, 1997.

[3] S. Rovnyak, S. Kretsinger, J. Thorp, D. Brown, Decision trees for real-time transient stability prediction. Power Systems, IEEE Transactions on, 9: 1417-1426, 1994.

[4] L. Wehenkel, M. Pavella, E. Euxibie and B. Heilbronn, Decision tree based transient stability method a case study. Power Systems, IEEE Transactions on, 9: 459-469, 1994.

[5] C. Cortes, V. Vapnik, Support-vector networks. Machine Learning, 20: 273-297, 1995.

[6] C. Bo-Juen, C. Ming-Wei, J. Chih, Load forecasting using support vector Machines: a study on EUNITE competition 2001. Power Systems, IEEE Transactions on, 19: 1821-1830, 2004.

[7] L. S. Moulin, S. A. da, M. A. El-Sharkawi and R. J. Marks, Support vector machines for transient stability analysis of large-scale power systems. Power Systems, IEEE Transactions on, 19: 818825, 2004.

[8] X. Wang, S. Wu, Q. Li and X. Wang, v-SVM for transient stability assessment in power systems. ISADS 2005. Proceedings, pp. 356-363, 2005. 
[9] L. S. Moulin, S. A. da, M. A. El-Sharkawi and R. J. Marks, Support vector and multilayer perceptron neural networks applied to power systems transient stability analysis with input dimensionality reduction. Power Engineering Society Summer Meeting, 2002 IEEE, 3:1308$1313,2002$.

[10] S. Tso, X. G., Y. Z. and L. L., An ANN-based multilevel classification approach using decomposed input space for transient stability assessment. Electric Power Systems Research, 46: 259-266, 1998.

[11] C.C. Chang, C.-J. Lin, LIBSVM : a library for support vector machines, 2001. http://www.csie.ntu.edu.tw/ cjlin/libsvm.

[12] I.H. Witten, E. Frank, Data Mining: Practical machine learning tools and techniques, 2nd editioned. Singapore: Elsevier, 2005.

[13] V.Vittal, (Chairman) Transient stability test systems for direct stability methods. IEEE Transaction on Power Systems, 7(1): 37-43, 1992. 